\title{
Progranulin mutations in Dutch familial frontotemporal lobar degeneration
}

\author{
Iraad F Bronner ${ }^{1,2,4}$, Patrizia Rizzu ${ }^{\star 1,2,4}$, Harro Seelaar ${ }^{3}$, Saskia E van Mil ${ }^{1,2}$, Burcu Anar ${ }^{1,2}$, \\ Asma Azmani ${ }^{3}$, Laura Donker Kaat ${ }^{3}$, Sonia Rosso ${ }^{3}$, Peter Heutink ${ }^{1,2}$ and John C van Swieten ${ }^{3}$ \\ ${ }^{1}$ Department of Human Genetics, Section Medical Genomics, VU University Medical Center and VU University, \\ Amsterdam, The Netherlands; ${ }^{2}$ Center for Neurogenomics and Cognitive Research, VU University Medical Center and \\ VU University, Amsterdam, The Netherlands; ${ }^{3}$ Department of Neurology, Erasmus MC University Medical Center, \\ Rotterdam, The Netherlands
}

Mutations in the progranulin (PGRN) gene have recently been identified in frontotemporal lobar degeneration with ubiquitin inclusions linked to chromosome 17q21. We report here the finding of two novel frameshift mutations and three possible pathogenic missense mutations in the PGRN gene. Furthermore, we determined the frequency of $P G R N$ mutations in familial cases recruited from a large population-based study of frontotemporal lobar degeneration carried out in The Netherlands. European Journal of Human Genetics (2007) 15, 369-374. doi:10.1038/sj.ejhg.5201772; published online 17 January 2007

Keywords: PGRN; FTLD; mutations; neurodegeneration

\section{Introduction}

The term frontotemporal lobar degeneration (FTLD) refers to an heterogeneous group of neurodegenerative disorders clinically characterized by progressive behavioral changes and cognitive dysfunctions, including executive and language functions. ${ }^{1}$ Sometimes, language impairment presents as an initial symptom sub-classifying this FTLD group into progressive nonfluent aphasia and semantic dementia. Additionally, the clinical picture can be complicated by motor symptoms such as motor neuron disease (MND) or parkinsonism. ${ }^{2}$

Two main pathological FTLD subtypes are recognized based on the presence of tau-positive inclusions (tauopathies) or tau-negative ubiquitin-positive neuronal inclusions (FTLD-U). ${ }^{3} \quad$ Characteristically the ubiquitin immunoreactive inclusions (ub-i) are observed in the

\footnotetext{
*Correspondence: Dr P Rizzu, Department of Human Genetics, Section Medical Genomics and Center for Neurogenomics and Cognitive Research, VU University Medical Center and VU University, Amsterdam, van der Boechorststraat 7, 1081 BT Amsterdam, The Netherlands. Tel: + 3120 5989961; Fax: + 3120 4448285; E-mail: p.rizzu@vumc.nl ${ }^{4}$ These authors contributed equally to this work.

Received 25 September 2006; revised 29 November 2006; accepted 30 November 2006; published online 17 January 2007
}

dentate gyrus of the hippocampus and in the superficial layers of the frontal and temporal cortex. ${ }^{4}$

A positive family history is found in approximately $40 \%$ of FTLD cases, and linkage studies have shown that FTLD is genetically heterogeneous with loci and genes identified on chromosomes 3 (FTD3), ${ }^{5} 9 \mathrm{p}^{6}{ }^{6} \mathrm{q}^{7}$ and $17 \mathrm{q}$ (FTDP- $17^{8}$ and FTDU- $17^{9,10}$ ). Recently, mutations in the PGRN gene were found in several families with FTDU-17. ${ }^{9,10}$ PGRN encodes a biologically active precursor glycoprotein described previously as a multifunctional growth factor involved in development, inflammation and wound repair. ${ }^{11}$

In the present study, we report the finding of two novel frameshift mutations and three possible pathogenic missense mutations in the progranulinv (PGRN) gene. In addition, we describe the genetic contribution of PGRN to FTLD in a series of familial cases recruited from a large cohort of FTLD patients.

\footnotetext{
Materials and methods

Patients

Three hundred and thirty-eight patients with FTLD (182 females and 156 males) with mean age at onset of
} 
$57.4 \pm 9.3$ years were identified in a genetic-epidemiological study in the Netherlands. The clinical diagnosis in all patients was established according to international consensus criteria. ${ }^{12}$ Clinical family history was positive in 166 patients (59\%) and among them DNA was available in 137 cases. Eighty-seven of these 137 patients came from independent families: 10 families presented MAPT mutations, ${ }^{13}$ two large families showed FTLD-U with definite linkage to chromosome 17q21-22, six smaller families had multiple $(>2)$ affecteds and 69 had two affecteds.

\section{DNA study}

The 13 exons of PGRN including intron/exons boundaries were amplified from genomic DNA by PCR and directly sequenced in both strands. Novel sequence variants were analyzed in a minimum of 380 chromosomes from healthy individuals of matched ethnicity.

\section{Immunohistochemistry}

Immunohistochemistry experiments were performed on eight available brains as described previously. ${ }^{14}$

\section{Results}

To determine the possible involvement of the newly found PGRN gene in our cohort, we systematically screened for mutations in 77 cases with positive family history of dementia consistent with autosomal dominant pattern of inheritance and with no MAPT and $C H M P 2 B$ mutations. The mean age at onset in this group was $59.3 \pm 9.1$ years.

We identified two novel frameshift mutations Ser82Valfs174X and Val411Sfr1X (Table 1) predicted to cause premature termination of the coding sequence likely leading to loss of functional PGRN protein similar to previous reports. One nonsense mutation (Gln125X) was also observed in a independently ascertained member of the 1083 FTLD-U family already described. ${ }^{10}$ Furthermore, we identified five novel coding sequence variants (three missense and two silent mutations), two intronic sequence changes in intron 2 and 7 and the previously reported missense mutation Gly414 Val. ${ }^{15}$ The frameshift mutations and the GGG93GGA, Thr182Met, Pro233His, CAC447CAT and Trp541Cys mutations were not found in controls; in contrast, the two intronic variants were also present in healthy individuals suggesting they are not pathogenic. Moreover, the GGG93GGA silent mutation was detected in co-occurrence with the Pro233His.

The Ser82Valfs174X mutation was found in a 69-year-old woman, member of the HFTD3 family previously linked to $17 q 21-22^{4}$ (Figure 1a). A large variation in age at onset (between 45 and 75 years) was observed between affected individual from this family.

Sequencing of 13 additional DNA samples from affected family members showed complete segregation of the mutation with the disease. The clinical symptoms in this family consisted of apathy, loss of initiative and interest, roaming behaviour and word finding difficulties. Two patients developed parkinsonism early in the course of the disease, which moderately responded to levodopa treatment. Signs of motor neuron disease were not observed. Extensive neuropsychological testing in six patients revealed impaired naming with normal comprehension of language.

The Val411Serfr1X mutation was identified in a 66-yearold woman, who presented with speech, and writing errors, and word finding difficulties. The patient showed social inappropriate behaviour and emotional bluntness. Magnetic resonance imaging showed asymmetric right-sided frontotemporal atrophy. The patient developed loss of initiative, and died from bronchopneumonia. Her mother and grandmother suffered from identical symptoms, whereas her uncle and a nephew were diagnosed as Pick's disease.

Table 1 PGRN mutations identified in FTLD patients and healthy control individuals

\begin{tabular}{|c|c|c|c|c|c|c|}
\hline Location & Genomic ${ }^{a}$ & Predicted $c D N A^{\mathrm{b}}$ & Protein ${ }^{\mathrm{c}}$ & Rs number & Patients $(N)$ & Controls $(N)$ \\
\hline Exon 2 & g.4407delC & c.243delC & Ser82ValfsX174 & & HFTD3 & - \\
\hline Intron 2 & g.4436G $>A$ & & & & 1 & 4 \\
\hline Intron 2 & g.4445G $>A$ & & & rs9897526 & 19 & 35 \\
\hline Exon 3 & g. $4559 G>A$ & c. $279 G>A$ & Gly93Gly & & 1 & - \\
\hline Intron3 & g. $4661 \mathrm{G}>\mathrm{C}$ & & & & - & 1 \\
\hline Exon 4 & g.5129C $>T$ & c. $592 \mathrm{C}>\mathrm{T}$ & $G \ln 125 X$ & & 1 & - \\
\hline Exon 5 & g. $5402 \mathrm{C}>\mathrm{T}$ & c. $545 \mathrm{C}>\mathrm{T}$ & Thr182Met & & 1 & - \\
\hline Exon 6 & g.5667>A & c. $698 \mathrm{C}>\mathrm{A}$ & Pro233His & & 1 & - \\
\hline Intron 7 & g. $6048 \mathrm{G}>\mathrm{A}$ & & & & 22 & 18 \\
\hline Exon 10 & g.6944_6945 delGT & c.1231_1232delGT & Val411SerfsX1 & & 1 & - \\
\hline Exon10 & g. $6954 \overline{\mathrm{G}}>\mathrm{T}$ & c. $1241 \bar{G}>T$ & Gly414Val & & 1 & 1 \\
\hline Exon 10 & g. $7054 \mathrm{C}>\mathrm{T}$ & c. $1341 \mathrm{C}>\mathrm{T}$ & His447His & & 1 & - \\
\hline Exon10 & g.6966G $>A$ & c. $1253 G>A$ & Arg418Gln & & - & 2 \\
\hline Exon11 & g. $7428 G>C$ & c. $1623 G>C$ & Trp541Cys & & 1 & - \\
\hline
\end{tabular}

${ }^{a}$ Numbering relative to NC_000017.9 Genbank Accession Number and starting at nucleotide 1.

${ }^{b}$ Numbering relative to NM_002087.2 starting at the ATG.

'Numbering relative to NP_002087.1. 

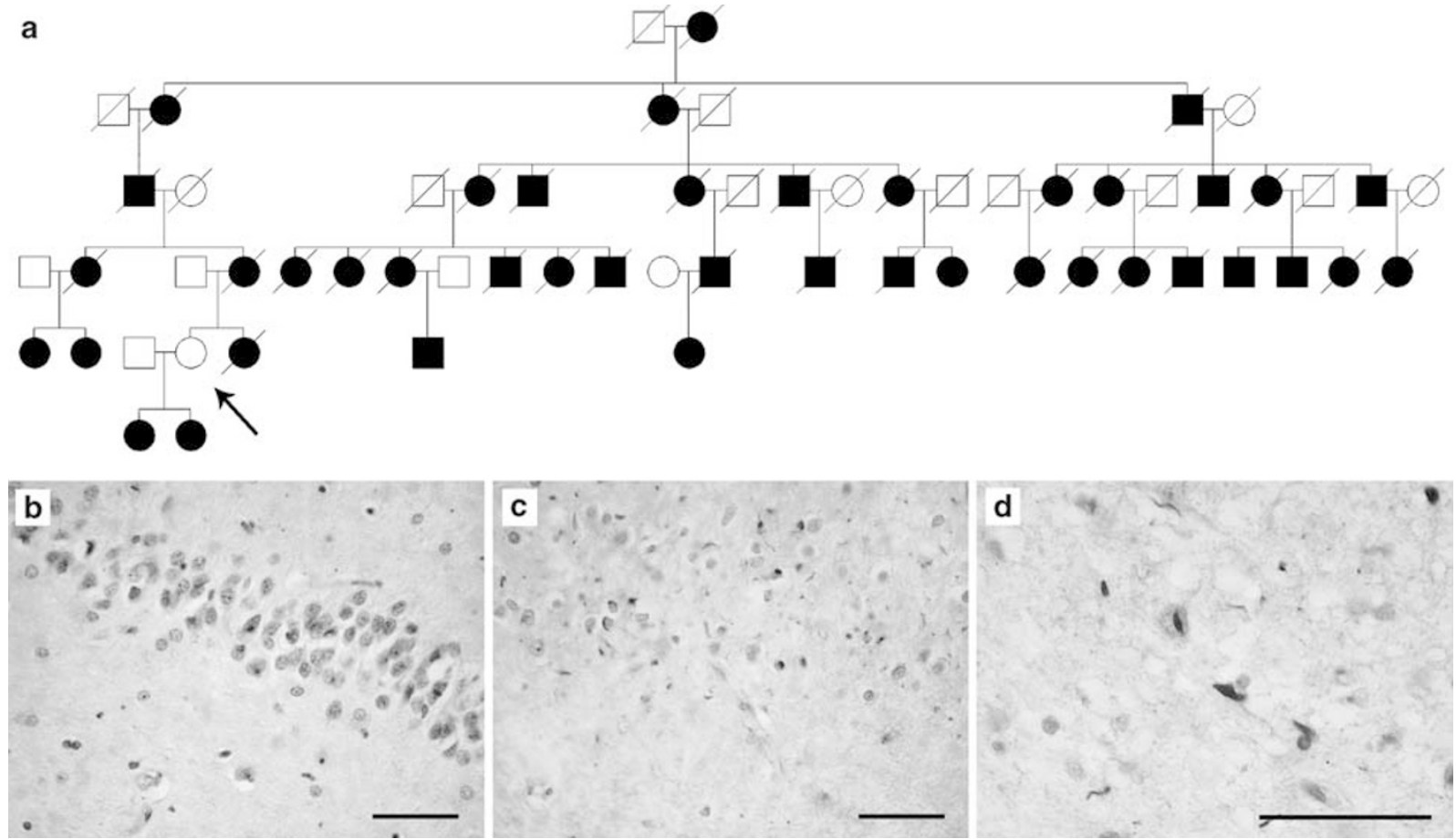

Figure 1 Pedigree and ubiquitin pathology (a). Pedigree of family HFTD3; only affected individuals are shown. This large family consists of 42 affected and 102 unaffected members. Arrow indicates a healthy carrier: a 72-year-old mother of two affected sisters who carried the mutation and did not have any cognitive complaints and behavioural changes as confirmed by reports of other family members and by neurological examination. (b-d) Ubiquitin staining; ubiquitin-positive neuronal cytoplasmatic inclusions in granular cells of the dentate gyrus (b) and in the superficial layers of the frontal neocortex (c) with ubiquitin-positive dystrophic neurites (c) in Ser82Valfs174X brain. Lentiform ubiquitin-positive neuronal intranuclear inclusion in one FTLD case with no PGRN mutations (d). Scale bars: $100 \mu \mathrm{m}$.

The Gln125X mutation was found in a 60-year-old woman, who came from the family 1083, described previously. ${ }^{10}$ She presented with memory problems and word findings difficulties.

Neuropathological examination showed ub-i in dentate gyrus, neocortex and/or striatum (Figure $1 \mathrm{~b}$ and c). Ubiquitin inclusions were also present in six additional brains from FTLD cases with no PGRN mutations with the distinct morphology and distribution pattern characteristic of FTLD-U type $2,{ }^{16}$ including several neuronal intranuclear inclusions in the frontal neocortex in one case (Figure 1d).

\section{Discussion}

The present study report the identification of three pathogenic (Val411Serfr1X and Ser82Valfs174X as novel) PGRN mutations that account for $\sim 4 \%$ of the independent familial FTLD cases.

Similar to previous PGRN studies the two novel mutations determine a frameshift, which results in the generation of premature termination codons. Eukaryotic cells are capable to detect and degrade transcripts harbouring premature signals for the termination of translation through the nonsense-mediated mRNA decay (NMD) pathway. Degradation of mutant mRNAs results in null alleles ${ }^{9,15}$ with loss of functional PGRN.

Several rare missense and silent mutations were detected in patients but not in controls. Segregation studies could not be performed in these cases, as DNA from affected family members was not available.

Although it cannot be excluded that these changes are benign variants as they are located in granulin domains each composed of 7,5 tandem repeats of highly conserved motifs of 12 cystein residues suggested to be functional redundant, ${ }^{15}$ several studies have shown that separate repeats may have alternative binding capacities and therefore different functions, ${ }^{17}$ highlighting the possibility that these variants are pathogenic. The Pro233 and the Trp541, in particular, are highly conserved among species (Figure 2a) and in the granulin domains (Figure 2b). Furthermore, previous reports have suggested these amino acids are essential for the proper folding of the protein. ${ }^{18-20}$ The Trp residue is likely involved in the hydrophobic packaging of the beta-sheet and substitution with the cys residue, which has the ability to form disulfide bridges, might affect PGRN 3D structure. The Pro residue is part of an antiparallel beta-sheet, and might be important for stacking multiple repeats, 


\section{a GRANULIN MOTIF II}

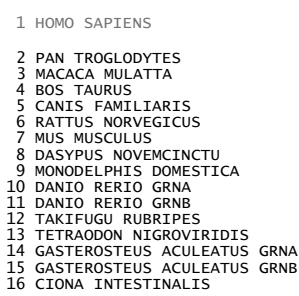

2 PAN TROGLODYTES

CANIS FAMILIARIS

MUS MUSCULUS

DANIO RERIO GRNA

TETRAODON NIGROVIR

15 GASTEROSTEUS ACULEATU

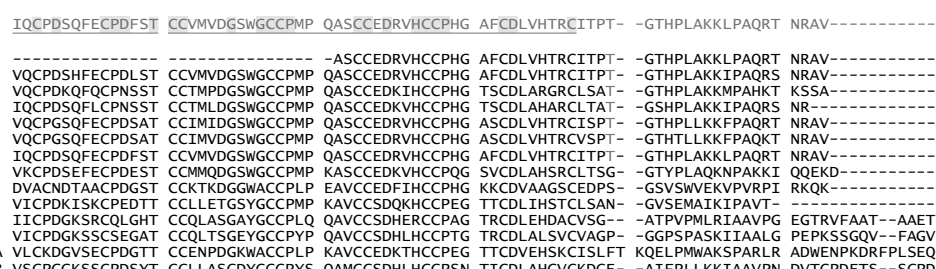

GRANULIN MOTIF III

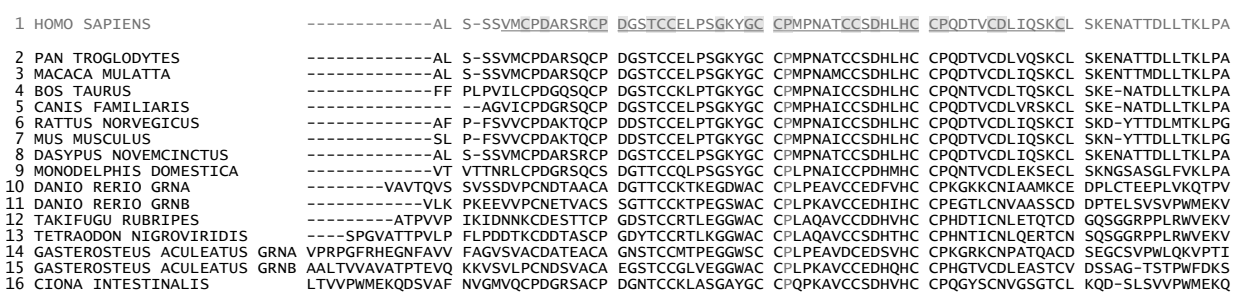

GRANULIN MOTIF V-a
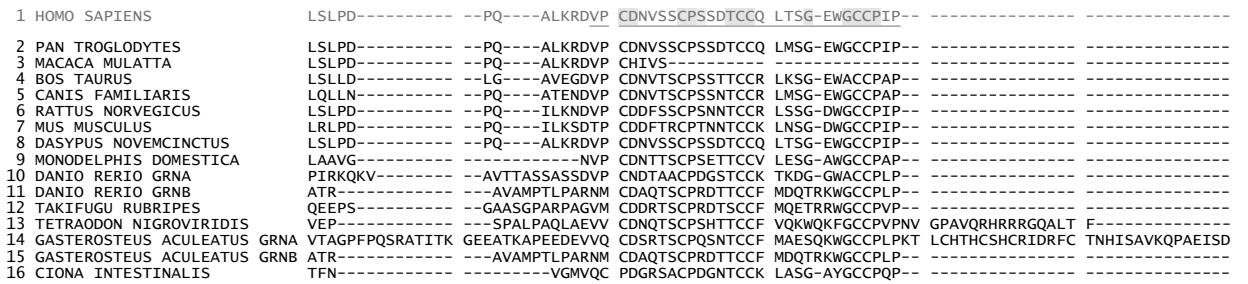

\section{GRANULIN MOTIF V-b}

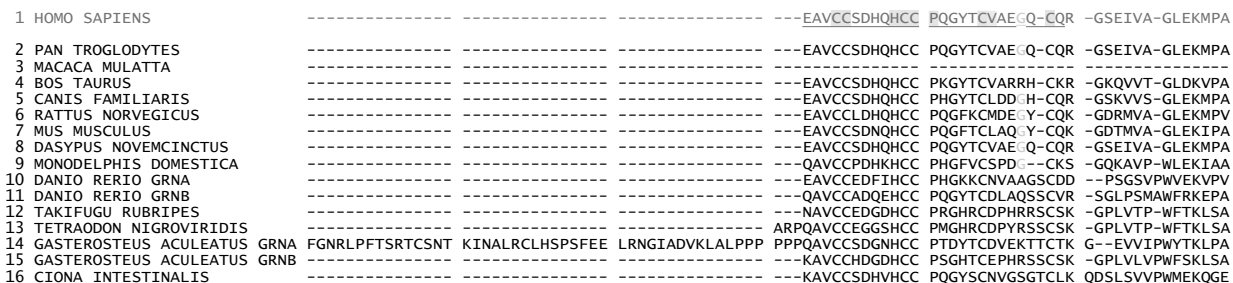

\section{GRANULIN MOTIF VII}
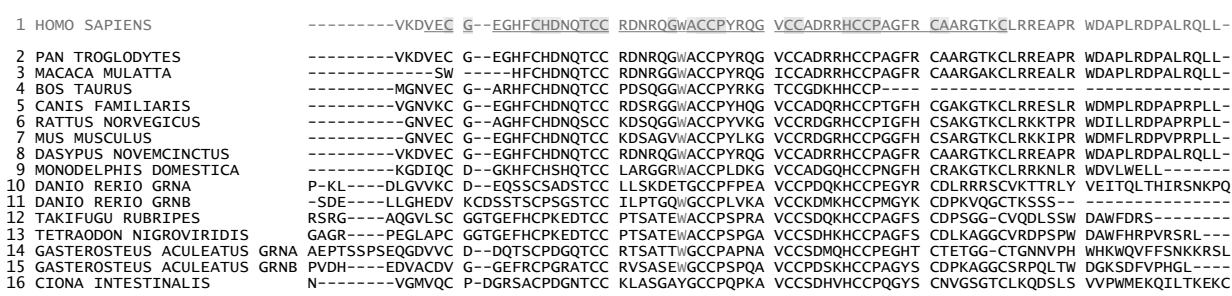

\section{b HUMAN PROGRANULIN}

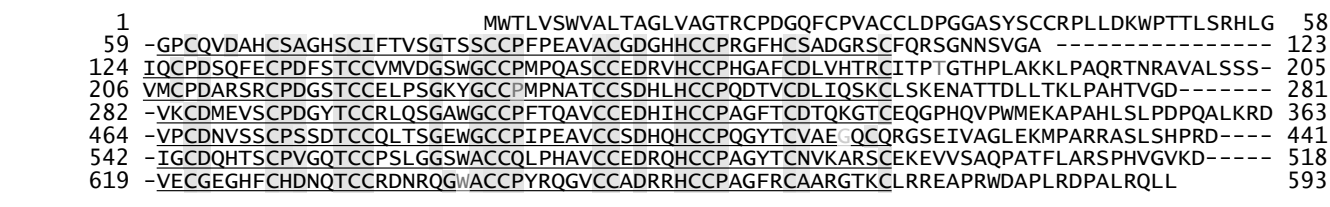

CONSENSUS --C-D----CPD--TCC----G-WGCCP-----CC-D--HCCP----CD--G--C---T----- 
necessary for the proper protein conformation. Therefore, his substitution with the His residue may also change PGRN 3D structure with consequences at functional level. The effect of the Thr182Met mutation is less clear since it is just outside the granulin motif. This amino acid is conserved between mammals and was not detected in controls.

Consistent with other PGRN studies, the clinical presentation in patients carrying the pathogenic mutations is characterized by a large variation in age at onset and by occurrence of symptoms of nonfluent aphasia, whereas semantic deficits were more often seen in patients with missense and intronic MAPT mutations within the same cohort. ${ }^{21}$ The HTFD3 family, in particular, shows a large variation in age at onset. The high variability is further confirmed by the presence of a 72-year-old healthy carrier. Our and other findings show that a significant proportion of patients remain unaffected until old age suggesting therefore an interplay of several genetic and/or environmental factors in the disease development.

The percentage of PGRN mutations detected in our familial FTLD cohort (up to $\sim 7 \%$ by including the two highly conserved missense mutations) is lower compared to the much higher frequency observed in other studies where PGRN mutations explain up to $\sim 25 \%$ of familial FTLD. ${ }^{10,15}$ The lower frequency of $P G R N$ mutations in our group might reflect differences in patients recruitment methods, as the MAPT mutations in the Belgian cohort account for only to $7 \%$ of all familial cases compared to $14 \%$ detected in this cohort. ${ }^{9,10,14}$ In addition, in the studies by Cruts et al. ${ }^{10}$ and Baker et al. ${ }^{9}$ a strong founder effect among probands carrying the IVSO $+5 \mathrm{G}>\mathrm{C}$ and Arg493X was observed, whereas we restricted our estimation of mutation frequency to independent patients only.

In addition, geographical differences in frequencies may also play a role, as seen in MAPT studies, and they cannot be ruled out until more reports will allow a better estimate of PGRN mutation frequency in familial FTLD.

In summary, mutations in PGRN explain only part of FTLD in our cohort and they are absent in $\sim 80 \%$ of cases including familial FTLD + MND as well as FTLD-U without MND strongly suggesting that we are only beginning to unravel the molecular pathways leading to FTLD and that additional genes contribute to the disease pathogenesis.

\section{Acknowledgements}

This study was supported by the Centre for Medical Systems Biology (CMSB), a centre of excellence approved by the Netherlands Genomics Initiative/Netherlands Organization for Scientific Research (NWO) and by the 'Hersenstichting Nederland' Project-Number 13F05(2).14 and 'Stichting Dioraphte'.

\section{References}

1 Neary D, Snowden JS, Gustafson L et al: Frontotemporal lobar degeneration - a consensus on clinical diagnostic criteria. Neurology 1998; 51: 1546-1554.

2 Forman MS, Farmer J, Johnson JK et al: Frontotemporal dementia: clinicopathological correlations. Ann Neurol 2006; 59: 952-962.

3 McKhann GM, Albert MS, Grossman M, Miller B, Dickson D, Trojanowski JQ: Clinical and pathological diagnosis of frontotemporal dementia: report of the Work Group on Frontotemporal Dementia and Pick's Disease. Arch Neurol 2001; 58: 1803-1809.

4 Rosso SM, Kamphorst W, de Graaf B et al: Familial frontotemporal dementia with ubiquitin-positive inclusions is linked to chromosome 17q2l-22. Brain 2001; 124: 1948-1957.

5 Skibinski G, Parkinson NJ, Brown JM et al: Mutations in the endosomal ESCRTIII-complex subunit CHMP2B in frontotemporal dementia. Nat Genet 2005; 37: 806-808.

6 Vance C, Al-Chalabi A, Ruddy D et al: Familial amyotrophic lateral sclerosis with frontotemporal dementia is linked to a locus on chromosome 9p13.2-21.3. Brain 2006; 129: 868-876.

7 Hosler BA, Siddique T, Sapp PC et al: Linkage of familial amyotrophic lateral sclerosis with frontotemporal dementia to chromosome 9q21-q22. JAMA 2000; 284: 1664-1669.

8 Hutton M, Lendon CL, Rizzu P et al: Association of missense and $5^{\prime}$-splice-site mutations in tau with the inherited dementia FTDP-17. Nature 1998; 393: 702-705.

9 Baker M, Mackenzie IR, Pickering-Brown SM et al: Mutations in progranulin cause tau-negative frontotemporal dementia linked to chromosome 17. Nature 2006; 442: 916-919.

10 Cruts M, Gijselinck I, van der Zee J et al: Null mutations in progranulin cause ubiquitin-positive frontotemporal dementia linked to chromosome 17q21. Nature 2006; 442: 920-924.

$11 \mathrm{He}$ Z, Bateman A: Progranulin (granulin-epithelin precursor, PC-cell-derived growth factor, acrogranin) mediates tissue repair and tumorigenesis. J Mol Med 2003; 81: 600-612.

12 The Lund and Manchester Groups: Clinical and neuropathological criteria for frontotemporal dementia. I Neurol Neurosurg Psychiatry 1994; 57: 416-418.

13 Rizzu P, van Mil SE, Anar B et al: CHMP2B mutations are not a cause of dementia in Dutch patients with familial and sporadic frontotemporal dementia. Am J Med Genet B Neuropsychiatr Genet 2006; 141: $944-946$.

14 Rosso SM, Kaat LD, Baks T et al: Frontotemporal dementia in The Netherlands: patient characteristics and prevalence estimates from a population-based study. Brain 2003; 126: 2016-2022.

15 Gass J, Cannon A, Mackenzie IR et al: Mutations in progranulin are a major cause of ubiquitin-positive frontotemporal lobar degeneration. Hum Mol Genet 2006; 15: 2988-3001.

16 Sampathu DM, Neumann M, Kwong LK et al: Pathological heterogeneity of frontotemporal lobar degeneration with

Figure 2 (a) Progranulin motif alignment between species. Human progranulin sequence is given in blue. Only progranulin motifs containing mutations are shown. Progranulin motifs are underlined. Conserved amino acids described by He and Bateman ${ }^{11}$ are turquoise and nonconserved amino acids are shown in yellow. The possible Thr182Met, Pro233His and Trp541Cis mutations are shown in red. The Gly414Val polymorphism is given in green. (b) Conservation of amino acids between progranulin motifs. The longest human progranulin isoform is shown. All human progranulin motifs are aligned. Conserved amino acids described by He and Bateman ${ }^{11}$ are turquoise and non-conserved amino acids are shown in yellow. Consensus depicts the consensus motif adapted from He and Bateman. ${ }^{11}$ The possible Thr182Met, Pro233His and Trp541Cys mutations are shown in red. The Gly414Val polymorphism is given in green. 
19 Tolkatchev D, Ng A, Vranken W, Ni F: Design and solution structure of a well-folded stack of two beta-hairpins based on the amino-terminal fragment of human granulin A. Biochemistry 2000; 39: 2878-2886.

20 Vranken WF, James S, Bennett HP, Ni F: Solution structures of a 30-residue amino-terminal domain of the carp granulin-1 protein and its amino-terminally truncated 3-30 subfragment: implications for the conformational stability of the stack of two betahairpins. Proteins 2002; 47: 14-24.

21 Neary D, Snowden J, Mann D: Frontotemporal dementia. Lancet Neurol 2005; 4: 771-780. 